Instituto Internacional de Investigación y Desarrollo Tecnológico Educativo INDTEC, C.A.

DOI: https://doi.org/10.29394/Scientific.issn.2542-2987.2020.5.15.2.44-66

OAI-PMH: http://www.indteca.com/ojs/index.php/Revista Scientific/oai

Artículo Original / Original Article

\title{
Comportamiento Mediático y Estrategias de aprendizaje informales en adolescentes ecuatorianos
}

\author{
Autores: Andrea Ximena Castaño Sánchez \\ Universidad Nacional de Educación, UNAE \\ andrea.castano@unae.edu.ec \\ Azogues, Ecuador \\ https://orcid.org/0000-0002-8787-0468 \\ Marcos Manuel Ibarra Núñez \\ Universidad Nacional de Educación, UNAE \\ marcos.ibarra@unae.edu.ec \\ Azogues, Ecuador \\ https://orcid.org/0000-0002-3534-9005 \\ Manuel Torres Mendoza \\ Universidad Estatal Península de Santa Elena, UPSE \\ mtorres@upse.edu.ec \\ Guayaquil, Ecuador \\ https://orcid.org/0000-0002-5844-657X
}

\section{Resumen}

La presente investigación indaga sobre el comportamiento mediático de jóvenes ecuatorianos entre 9 y 16 años en su realidad cotidiana interactuando con diversos medios digitales, con el fin de identificar sus estrategias de aprendizaje informal. Para esta investigación, se siguió un estudio etnográfico en menor escala y de corto tiempo. Los resultados demostraron el uso que hacen los jóvenes, se traslada hacia la utilización de videos, como tutoriales, para aprender y también para la diversión, el uso de las redes sociales con propósitos en ocasiones informativos, y la cultura de participación en los medios sociales que refleja la realidad y el contexto en el que se desarrollan. Se describen los perfiles de jóvenes en contextos cambiantes y evidencian las competencias identificadas previamente, de las que son representativas como parte de las estrategias informales y la capacidad para autorregular el consumo mediático, capacidad para buscar fuentes de información, capacidad para autogestionar los recursos y el tiempo, capacidad para usar recursos transmedia, capacidad para analizar contenido multimedia, y utilizar recursos offline. Este estudio es parte de un proyecto transnacional que tiene por objetivo la observación de las prácticas juveniles en relación con la cultura participativa.

Palabras clave: aprendizaje informal; adolescente; aprendizaje; habilidad.

Cómo citar este artículo:

Castaño, A., Ibarra, M., \& Torres, M. (2020). Comportamiento Mediático y Estrategias de aprendizaje informales en adolescentes ecuatorianos. Revista Scientific, 5(15), 44-66, e-ISSN: 2542-2987. Recuperado de: https://doi.org/10.29394/Scientific.issn.2542-2987.2020.5.15.2.44-66

Fecha de Recepción: 18-09-2019
Fecha de Aceptación: 21-12-2019
Fecha de Publicación: 05-02-2020 


\title{
Media behavior and informal learning strategies in Ecuadorian adolescents
}

\begin{abstract}
This research investigates the media behavior of young Ecuadorians between 9 and 16 years old in their daily reality interacting with various digital media, in order to identify their informal learning strategies. For this investigation, a smaller and short-term ethnographic study was followed. The results demonstrated the use that young people make, moves towards the use of videos, as tutorials, to learn and also for fun, the use of social networks for informational purposes, and the culture of participation in social media It reflects the reality and context in which they develop. The profiles of young people in changing contexts are described and evidence of the previously identified competencies, of which they are representative as part of informal strategies and the ability to self-regulate media consumption, ability to search for sources of information, ability to self-manage resources and time, ability to use transmedia resources, ability to analyze multimedia content, and use offline resources. This study is part of a transnational project whose objective is the observation of youth practices in relation to participatory culture.
\end{abstract}

Keywords: informal learning; teenagers; learning; skill.

\section{How to cite this article:}

Castaño, A., Ibarra, M., \& Torres, M. (2020). Media behavior and informal learning strategies in Ecuadorian adolescents. Revista Scientific, 5(15), 44-66, e-ISSN: 2542-2987. Recovered from: https://doi.org/10.29394/Scientific.issn.2542-2987.2020.5.15.2.44-66

Date Received: 18-09-2019
Date Acceptance: 21-12-2019
Date Publication: 05-02-2020 


\section{Introducción}

Este trabajo es producto del proyecto Educación Transmedia. Competencias transmedia y estrategias informales de aprendizaje de los adolescentes, llevado a cabo en la Universidad Nacional de Educación de Ecuador (UNAE) y cuya investigación se desarrolló en escuelas de la zona 6 de Ecuador.

El comportamiento de los jóvenes en la sociedad de la información y la comunicación se presenta con nuevas formas de aprender y mayormente en la interacción en línea o el mundo de la virtualidad. Exponen Livingstone y Sefton-Green (2016): que desde los últimos veinte años, la retórica de la "Edad Digital" en cuanto al llamado sobre los avances en tecnologías, redes de información y nuevos medios, ha ocasionado una reestructuración en la sociedad, las escuelas, las familias y en general (pág. 3). Y para Lerma, Cortés y Barrera (2016): las tecnologías están facilitando un contexto donde surgen nuevas formas de ser, relacionarse, movilizarse, informarse y participar de alguna forma, los jóvenes han crecido interactuando con medios digitales, en mayor o menor medida dependiendo del contexto (pág. 741). En esta misma línea, García-Galera y Valdivia (2014): asumen que esta interacción ha llevado a formas de hacer, conocer y ser diferente a las generaciones anteriores, por medio de acciones claves que caracterizan a un joven que consume y produce medios, es decir que compone, comparte y difunde información promoviendo lo que se puede denominar una "cultura participativa" (pág. 11). Por lo tanto, estos aspectos cambiantes son de gran interés en el momento actual porque inducen a investigaciones sobre la forma cómo viven los jóvenes la escuela hoy, sus comportamientos recreativos, y qué puede estar en déficit o en construcción en sus interacciones (digitales), en sus contextos informales y formales educativos.

Por consiguiente, los jóvenes sujetos a esta investigación pueden estar desarrollando aprendizajes informales, que les permitan construir un puente 
entre lo que ellos por si mismos han desarrollado en la interacción diaria en la era digital a través de herramientas que ellos mismos descubren a diario, y el desarrollo de aprendizajes formales en sincronía con el uso de estrategias didácticas. Este accionar de los jóvenes es el objeto de estudio de esta investigación que motiva a la búsqueda de patrones de comportamiento que informen sobre la forma cómo interactúan con diversos medios digitales y las estrategias que emplean para hacer perdurables los conocimientos $y$ aprendizajes adquiridos en este tipo de interacciones y poder llevarlos a la educación formal, ya que como dice Camacho (2018): "los estudiantes nativos digitales en esta era tecnológica actúan semánticamente diferente a los profesores inmigrantes digitales y análogos" (pág. 23); lo que crea una brecha entre la manera de enseñar de los docentes y la de aprender de los jóvenes.

\section{Desarrollo}

\subsection{Educomunicación y cultura participativa como elemento clave en los aprendizajes}

La educomunicación en la Escuela implica una revisión integral al currículo escolar de forma que promueva la adaptación curricular tecnológica con las necesidades y motivaciones de aprendizaje de los estudiantes, y a su vez permita una alfabetización de la Educación mediática "Media literacy", en el alumnado y el profesorado. Según Fernández, Blasco-Duatis y CaldeiroPedreira (2016a): esto sería posible por medio de estrategias didácticas apoyadas en las nuevas formas de aprender de los adolescentes en sus propios espacios informales, que el docente actual debe utilizar para fomentar el diálogo y la construcción de conocimiento dentro de los espacios educativos formales (pág. 27).

La educomunicación favorece, además, al aprendizaje que permite la navegación a través de relatos transmedia. Desde esta mirada entendemos que la educación mediática está basada en las representaciones, la Media 
comunicación está basada en símbolos y signos que deben ser codificados y decodificados. En esta línea, Masterman (2010): ha estudiado las formas cómo diversos contenidos están siendo representados, simbolizados y empacados por los medios; el concepto central de la educación a través de los medios es la de representación. Los medios no reflejan el mundo, pero si lo representan (pág. 19).

En este orden de ideas, Aparici y Osuna (2013): expresan que la cultura participativa se ha caracterizado por el auge de la Web 2.0 que ha posibilitado un modelo de interacción entre las personas en condiciones de igualdad que promueve la libre expresión, la creatividad y la puesta en común de creaciones propias y colectivas (pág. 138). En este mismo sentido, Pereira, Vaz y Tognetti (2017a): manifiestan la posibilidad de poder compartir nuestras experiencias diarias a través de diferentes dispositivos y plataformas lo que ha hecho que se modifiquen las formas de consumo y la creación de contenido en red, por lo que destaca en la cultura de la participación: el fuerte apoyo para crear y compartir creaciones personales con los demás, la tutoría informal como forma de pasar el conocimiento experto al más novato, la valoración de las contribuciones, la conexión social entre diversos miembros de una comunidad, la confianza puesta en la contribución voluntaria, donde todas las personas tienen voz y es válida en las comunidades virtuales (pág. 11). Esto se extiende a los aprendizajes informales y formales transmedia que son los objetos de este estudio.

Paralelamente, Pereira, Vaz y Tognetti (2017b): es de este modo que la cultura participativa va tomando forma de acuerdo con los usos dados a las redes sociales, como medio principal para reflejar los comportamientos tanto de consumo como de producción de los jóvenes actuales (pág. 12). Es por medio de estas prácticas que la participación social y cívica es reflejada desde la realidad hacia la virtualidad, que ha ido a la par con una revolución en la forma en la que la sociedad se expresa. 
En esta forma, Jenkins (2011): expone sobre la interacción con los medios digitales es imperativa, no es posible la existencia de un texto sin un mínimo de interacción, por parte del receptor (párr. 17). Por lo tanto, también hay un espectador mediático que de alguna forma presenta un rol activo, de forma que siempre es posible un trabajo mental semiótico e interpretativo. En consecuencia, la cultura participativa se expresa en los jóvenes cuando a través de sus contribuciones son parte de una comunidad que ellos mismo eligen, es así, que pueden ser autónomos de forma que sus comportamientos en la vida real se reflejan en la virtual, y en la forma como interpretan un mismo mensaje desde diversos medios, propiciando así entre ellos y sin ser conscientes de ello, los espacios transmedia.

\subsection{Aprender a través del transmedia}

Según Jenkins, Ford y Green (2015a): el transmedia concebido desde las narrativas digitales parte de la forma como se cuenta una historia o una experiencia, desde múltiples plataformas y formatos, usando las actuales tecnologías digitales (pág. 88). Para Scolari (2013a): el concepto transmedia puede ser analizado desde diversos enfoques, la intertextualidad transmediática, la narrativa transmedia, igualmente que los mundos de narración transmedia (pág. 19).

Todos los conceptos anteriores de análisis ofrecen diferentes perspectivas que contribuyen a que muchos más jóvenes de diferentes lugares del mundo estén empezando a concretar usos cada vez más diversos de las narrativas transmedia, que han permitido que ellos mismos se adapten a formas únicas de comunicar desde diferentes culturas, comunidades, y así mismos articulados a escenarios progresivamente más diversos. En este sentido, las características de estas prácticas están sucediendo por medio de la influencia de diversos factores y tendencias presentes en los diversos contextos. Algunos estudios han podido realizar un análisis desde los usos del 
transmedia hasta los tipos de alfabetismos que en consecuencia se producen.

En adición a Alper y Herr-Stephenson (2013): ofrecen una perspectiva sobre el transmedia en su reporte "Aprendiendo a través del juego transmedia" (pág. 367); donde hacen énfasis en el transmedia como un recurso de aprendizaje en varios contextos a través del juego y la experimentación, mediante la expresión y la participación, por medio del compromiso cultural, que respeta los pensamientos y sentimientos de quienes crean. De esta forma pueden emerger varios tipos de alfabetización en el proceso de creación de proyectos transmedia: multimodal, visual, informacional, en jugabilidad (solución de problemas y pensamiento estratégico). En consecuencia, se hace un mapeo y presenta seis tendencias marcadas en los jóvenes en términos de creación y consumo de contenido mediático: 1). Períodos cortos de atención, 2). acercamiento de intereses, 3). serenidad social, 4). curaduría comunitaria, 5). remixes en contenido, y 6). narrativas emergentes.

Además, estas tendencias se relacionan en un marco de aprendizaje llamado Aprendizaje Conectado-Connected Learning que se rige bajo los principios de aprendizaje: 1). dirigidos por el interés, 2). apoyado entre pares, 3). orientados académicamente. Este tipo de marco es un referente para propuestas de estrategias didácticas basada en la participación de aprendices y estudiantes y el desarrollo de habilidades para conectar personas, recursos y contenidos. Esto implica un cambio educacional que supone desarrollar tipos de habilidades a través de acciones concretas durante el desarrollo de proyectos transmedia y diversas acciones trasladadas a diferentes actividades de aprendizaje de conectar, compartir, analizar, evaluar, aplicar, personalizar, incluir, difundir, conocer, computar y construir.

Lo que es imprescindible es una formación crítica en los medios, en la misma cultura participativa, que permita a los menores enfrentarse a los retos mediáticos actuales y futuros. 


\subsection{De las estrategias de aprendizaje informal a las formales}

Las estrategias de aprendizaje informal se representan por los comportamientos habituales desarrollados por la exposición al entorno inmediato y que se desencadenan en aprendizajes. Siguiendo a Contreras y León (2019): a menudo, estos comportamientos permean en los contextos académicos, a pesar de lo disfuncional que pueda provocar el continuo acceso a Internet en los jóvenes, al encontrarse en una etapa crítica de definición de la propia identidad que puede llevar a un uso adictivo, si no es asesorado adecuadamente por familiares y tutores (pág. 12).

De manera que Jenkins, Ford y Green (2015b): en su libro titulado Cultura Transmedia, hace una crítica amplia sobre la forma como la tecnología está cambiando la cultura, de esta forma analiza las interacciones con diversos dispositivos, plataformas, y sobre todo los cambios que están provocando la sobreexposición a la información en Internet, permitiendo que la aparición de múltiples formatos y medios electrónicos y a su vez la redefinición de los comportamientos mediáticos (págs. 17-23).

Los jóvenes, quienes tienen la facilidad para navegar entre dispositivos e información, desarrollan ciertas estrategias de aprendizaje informal a medida que están insertos en una cultura participativa y de convergencia digital. Así mismo, Scolari (2017a), dice sobre el Proyecto Transliteracy, base para este estudio:

En el proyecto TRANSLITERACY, se identificaron seis modalidades de estrategias de aprendizaje informal (aprender haciendo, resolviendo problemas, imitando, jugando, evaluando y enseñando) que se interceptan con cuatro áreas principales (contenido, tiempo, espacio y relaciones) que contienen una serie de categorías y oposiciones (pág. 57). 
A continuación, se presentan en la tabla 1, estas 6 modalidades:

Tabla 1. Modalidades de estrategias de aprendizaje informal.

\begin{tabular}{|l|l|}
\hline \multicolumn{1}{|c|}{$\begin{array}{c}\text { Estrategias de } \\
\text { aprendizaje }\end{array}$} & \multicolumn{1}{c|}{ Descripción } \\
\hline Aprender haciendo & $\begin{array}{l}\text { Poner en práctica un grupo de actividades relacionadas } \\
\text { con la habilidad que desea adquirir (conlleva al ensayo y } \\
\text { error, experimentación). }\end{array}$ \\
\hline Solución de problemas & $\begin{array}{l}\text { Enfrentarse a un problema o suceso que le motivan a } \\
\text { adquirir la habilidad que necesita. }\end{array}$ \\
\hline Imitación/simulación & $\begin{array}{l}\text { Habilidad para auto-manejar recursos, tiempo, su propia } \\
\text { identidad, sentimientos y emociones. }\end{array}$ \\
\hline Jugar & $\begin{array}{l}\text { Adquisición de habilidades al involucrarse en ambientes } \\
\text { de jugabilidad. }\end{array}$ \\
\hline Evaluar & $\begin{array}{l}\text { Adquisición de habilidades al examinar su propio trabajo, } \\
\text { o ser evaluado por los demás. }\end{array}$ \\
\hline Enseñar & $\begin{array}{l}\text { Adquisición de habilidades al transmitir o demostrar } \\
\text { conocimientos a otros. }\end{array}$ \\
\hline
\end{tabular}

Fuente: Adaptado del documento de Scolari (2017).

Estas estrategias pueden desencadenar en aprendizajes por medio del Transmedia y el Multimedia, a partir de acciones específicas que dan como resultado una construcción grupal de conocimiento. Además, el hecho de que el joven sea consciente de estas estrategias permitiría el desarrollo de competencias transmedia que se desarrollan en un entorno de cultura participativa, de forma, que las mismas se pueden valorar de acuerdo con el resultado o producto de un individuo al compartir, crear, comentar, criticar y posicionarse frente a ese contenido mediático, de esta forma hay una conexión social y un tipo de aprendizaje informal donde un cierto tipo de conocimiento es desarrollado.

Adicionalmente, desde el proyecto Transliteracy se ha hecho una revisión a la serie de habilidades, identificados previamente por Jenkins, a partir de la identificación de comportamientos de los jóvenes como son: La apropiación como la habilidad para mezclar contenido digital; Multitarea la habilidad de cambiar la concentración en diversos detalles de un ambiente; Cognición distribuida, la habilidad de interactuar significativamente con 
diversas tareas; Inteligencia colectiva: la habilidad de obtener conocimiento desde otros para una tarea común; Juicio: habilidad para evaluar la credibilidad de diversas fuentes de información; Navegación transmedia: la habilidad de seguir una historia desde múltiples medios; Trabajo en red, la habilidad para buscar, sintetizar y diseminar información; Negociación la habilidad para moverse en diversas comunidades y discernir respecto de múltiples perspectivas; Visualización: habilidad para interpretar y crear datos y representaciones para expresar una idea o identificar tendencias.

De las habilidades identificados por este primer estudio, han evolucionado en el más reciente, hacia 9 dimensiones de producción, gestión de contenido, autogestión, gestión social, ejecución, tecnología y medios, narrativas y estética, ideología y ética, prevención de riesgos. De cada una de estas dimensiones el estudio las clasifica en 44 habilidades de segundo nivel y 190 habilidades específicas relacionadas con cada dimensión y que fueron el resultado del estudio consultando a expertos alrededor del mundo. Para el presente estudio, se realizó una clasificación que surgió de la investigación y se relaciona con algunas de las habilidades mencionadas anteriormente, se presenta en detalle en la sección de resultados.

De acuerdo con Fernández, Blasco-Duatis y Caldeiro-Pedreira (2016b): en el reporte Erasmus sobre el transmedia, se muestra como una nueva narrativa que comprende la educación y comunicación hacia el futuro, expone los resultados de la visión de la comunidad en relación con las tecnologías educativas digitales y su implementación en los centros educativos (pág. 7275). Donde concluye sobre una propuesta hacia una visión integradora en la ecología de medios, que promueve niveles más profundos de participación mediante el acceso a nuevas fuentes de información, mediante herramientas que relacionan la teoría con la práctica. En este contexto, Scolari (2013b): complementa que favorezcan la reflexión crítica a través de las tecnologías y las narrativas transmedia, así como lo ha demostrado con su evolución (pág. 
1420).

Este tipo de pensamiento crítico puede desarrollarse desde la visión de los medios como símbolos que deben ser decodificados. De esta forma, se puede enseñar acerca de los medios más efectivamente; no solo a través de un enfoque centrado en los contenidos, pero si a través de la aplicación de un marco conceptual de análisis que permita darle sentido. Aprendizajes movidos por el interés, apoyado entre pares, y orientados académicamente. Para Fernández, Blasco-Duatis y Caldeiro-Pedreira (2016c): es de esta forma, que la importancia del transmedia en las practicas digitales del conjunto de la comunidad educativa se puede convertir en nuevas realidades educativas en la comunidad pedagógica (pág. 75). El uso correcto que se pueda hacer de las redes sociales debido a su popularidad entre los jóvenes ha hecho repensar su utilización desde los contextos educativos formales, de forma que sea un medio adicional en la docencia con el objetivo de facilitar el proceso de aprendizaje, por medio de la interacción que se puede dar más allá de las limitaciones de espacio y tiempo de las aulas escolares.

Al respecto, Castells (2001): resalta como esa capacidad de aplicar lo aprendido, está bien influenciada por una sociedad que es desigual y que a su vez ha cambiado hacia una sociedad red, donde las relaciones de trabajo cambian siguiendo características sistémicas de los negocios en línea (pág. 18).

Respeto a lo anterior, es necesario promover una formación del profesorado para que favorezca la integración de una ecología de medios en la Escuela. En este sentido, las narrativas transmedia ofrecen la posibilidad de contar una única historia a partir de partes generadas desde diversos medios, lenguajes o plataformas, pero que se relacionan entre sí.

Promover las iniciativas de producción educativa transmedia desde espacios en la Web que permitan compartir los contenidos generados por los alumnos, de forma también que el alumnado pueda participar en la creación 
de nuevos medios. El docente debe estar capacitado para crear narrativas digitales, que puedan ser comunicadas desde diversas plataformas, por ejemplo, videos, imágenes, videojuegos educativos, podcasts, incluyendo los materiales concretos representados por pinturas, dibujos, íconos, material reciclable etc.

\section{Metodología}

La presente propuesta docente surge del proyecto de investigación Educación Transmedia: Competencias transmedia y estrategias informales de aprendizaje de los adolescentes financiado por la Universidad Nacional de Educación (UNAE), como parte de un proyecto trasnacional que tiene por objetivo la observación de las prácticas juveniles en relación con la cultura participativa. De acuerdo con Jordan (2016): representa un estudio etnográfico en menor escala y de corto tiempo. caracterizada por la indagación sobre las estrategias de aprendizaje informales que surgen desde la interacción cotidiana de los jóvenes con las redes sociales, los contenidos digitales, plataformas y el transmedia (pág. 12); este método de investigación fue elegido porque permitiría estudiar prácticas puntuales sobe el Qué, Cómo, y Para qué los jóvenes ecuatorianos interactúan con las transmedia digital. Participaron 136 estudiantes de los niveles $8^{\circ}$ de Educación General Básica (EGB) y $3^{\circ}$ de Bachillerato General Unificado (BGU).

En el marco del proyecto se utilizaron dos Instituciones Educativas de la de la Zona 6 del sur ecuatoriano, una en Cuenca y otra en Azogues, una rural y otra urbana, una fiscal y otra fiscomisional. Los datos se recolectaron a través de las siguientes técnicas: Observación participante en diferentes momentos de la investigación:

- Un (1) Cuestionario de uso mediático por parte de los estudiantes.

- Cuatro (4) Talleres de cultura participativa. 
- Cuatro (4) Talleres de videojuegos.

- Diecinueve (19) Entrevistas individuales en profundidad.

- Diecinueve (19) Diarios de uso de consumo mediático.

El análisis presentado en este estudio, se enfoca en los aspectos de la cultura participativa. El taller de cultura participativa, ha consistido en actividades relacionadas con la búsqueda de información, creación de contenidos mediáticos y su distribución en plataformas colaborativas, con el objetivo de entender y analizar cómo los adolescentes están adquiriendo habilidades y competencias transmedia fuera de la escuela. Para el análisis cualitativo de los datos se emplea el Software NVivo 11. Primero, se identifican las categorías que proceden del análisis de las entrevistas en relación con: ¿qué hacen? ¿cómo lo hacen? ¿cómo aprenden? y ¿cómo comparten? los adolescentes en entornos digitales. Posteriormente, de estas categorías se derivan otras subcategorías como se describe en el siguiente apartado.

\section{Análisis y resultados}

El análisis presentado a continuación se dio a la luz a través de las estrategias de aprendizaje emergente, de acuerdo con las categorías y la exploración realizada a 8 entrevistas escogidas de 19, hechas a estudiantes seleccionados por su activa participación en los talleres de cultura participativa. A continuación, se presentan las categorías y subcategorías de acuerdo con la codificación y el número de registros obtenidos para cada una de acuerdo con el Proyecto Transmedia Literacy: ¿Qué haces?: 648 registros: ¿Con qué lo haces?: 177 registros; ¿Por qué lo haces?: 3 registros; ¿Cómo aprendiste a hacerlo?: 61 registros; otros: 65 registros. Se debe tener en cuenta que cada una de estas categorías incluye sus propias subcategorías anidadas y que están dentro de esta misma codificación. 
El uso del Software de análisis cualitativo Nvivo 11, nos ha permitido aplicar algunos datos cuantitativos, basándose en el conteo de palabras con mayores registros en el conjunto de cada uno de los nodos de acuerdo con la codificación anterior. En la figura 1, se presentan las palabras más frecuentes del nodo presentado en una nube de palabras para la codificación 1: ¿Qué haces?. En ella se hace visible que además de YouTube (350 registros en las entrevistas) también se presenta Facebook (371 registros) y las palabras que aluden a lo que hacen los estudiantes (compartir: 389, información: 1293 registros, hacer: 609 registros, seguir: 102 registros), con las personas que se relacionan (amigos: 319 registros) y lo que utilizan (videos: 255 registros, canciones: 202 registros, juegos: 121 registros, plantillas: 10 registros, publicaciones: 285 registros).

Igualmente, para la codificación 2: ¿Con qué lo haces?, se presenta en forma de nube de palabras en la Figura 2, la frecuencia de las 50 palabras más utilizadas. Este análisis arroja las redes sociales utilizadas (Facebook: 207 registros, Twitter: 36 registros, YouTube: 207 registros, Snapchat: 8 registros, WhatsApp: 66 registros, Instagram 51 registros, Messenger: 55 registros) por medio del computador: 45 registros y el dispositivo móvil celular: 113 registros, con videos: 94 registros y otras aplicaciones: 84 registros.

El análisis realizado para la codificación 3: ¿Cómo lo haces?, es de especial interés para inducir sobre las estrategias de aprendizaje informal, en este sentido, se detalla cada una de las acciones con los registros en que aparecen en las entrevistas seleccionadas (descargar: 12, hacer: 31, decir; 9 , jugar: 9, comentar: 9, ver y aprender: 4) Objeto de las anteriores acciones aparece también la palabra: videos. 
Figura 1. Nube de palabras de la categoría: ¿Qué haces? ¿Con qué lo haces? ¿Cómo lo haces?
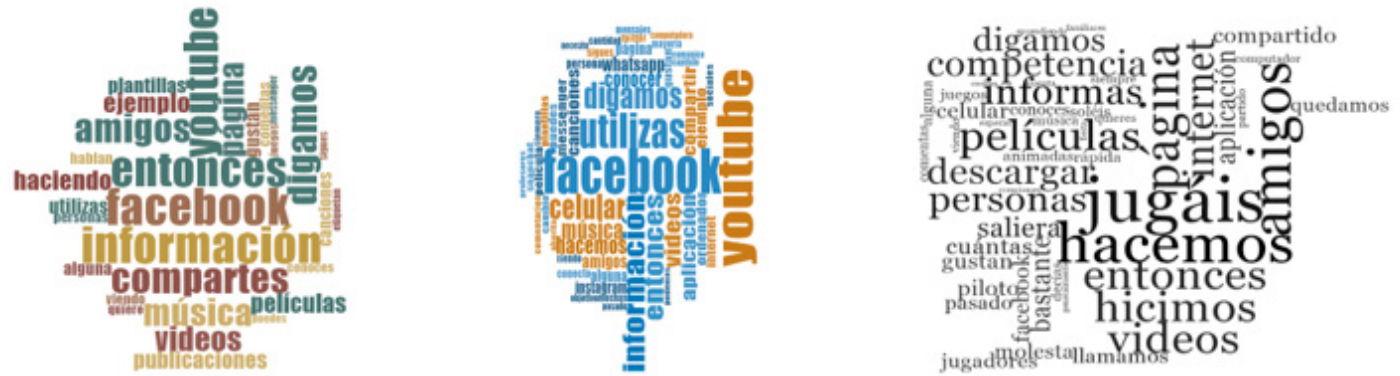

Fuente: Los Autores (2019).

A partir del análisis realizado de las codificaciones y subcodificaciones se han establecido las competencias transmedia obtenidas, que se muestran en la tabla 2 y tabla 3. La codificación de estas habilidades surgió de la relación con las estrategias informales utilizadas por los jóvenes ecuatorianos y evidenciadas en sus discursos.

Tabla 2. Competencias transmedia de los adolescentes ecuatorianos de la muestra y ejemplos de testimonios recogidos.

\begin{tabular}{|c|l|}
\hline Competencias Transmedia & \multicolumn{1}{c|}{ Testimonios } \\
\hline $\begin{array}{c}\text { Capacidad para usar diferentes } \\
\text { dispositivos, aplicaciones y buscadores. }\end{array}$ & $\begin{array}{l}\text { Ej. [Entrevista]: Si, por lo general tengo } \\
\text { prendido la Tv, la computadora y mi celular, } \\
\text { en la televisión pongo Tele hit, que dan } \\
\text { solo música, veo Facebook y mi celu para } \\
\text { WhatsApp y Snapchat y así. }\end{array}$ \\
\hline $\begin{array}{c}\text { Capacidad para buscar contenidos } \\
\text { audiovisuales, mediáticos mediante } \\
\text { diversas buscadores y plataformas o apps. }\end{array}$ & $\begin{array}{l}\text { Ej. [Entrevista]: En el buscador de YouTube } \\
\text { abre otra pestaña de Google y busca } \\
\text { convertidor mp3 escoge la opción } \\
\text { convertido YouTube a mp3, copia el link del } \\
\text { video y lo pega para descargar y lo } \\
\text { comparte en Google. }\end{array}$ \\
\hline $\begin{array}{c}\text { Capacidad para gestionar las fuentes de } \\
\text { información con base en información } \\
\text { relacionada en gustos de entretenimiento } \\
\text { mediante Internet. }\end{array}$ & $\begin{array}{l}\text { Ej. [Entrevista]: Cuando algo me interesa } \\
\text { de verdad, ahí lo busca hasta encontrarlo o o } \\
\text { guardo el video o lo comparto. }\end{array}$ \\
\hline
\end{tabular}

Fuente: Los Autores (2019). 
Tabla 3. Competencias transmedia de los adolescentes ecuatorianos de la muestra y ejemplos de testimonios recogidos.

\begin{tabular}{|c|c|}
\hline $\begin{array}{l}\text { Capacidad para autorregular el } \\
\text { consumo mediático por medio } \\
\text { del pensamiento secuencial. }\end{array}$ & $\begin{array}{l}\text { Ej. [Entrevista]: ...la mayoría de las veces, a veces sólo } \\
\text { las escribo en hojas sueltas, así. Como que esto de aquí } \\
\text { me ayuda a generar un poco más, digamos, lo que sería } \\
\text { la imaginación y puedo crear distintos, o sea } \\
\text { imaginarme distintos mundos; así mismo puedo estar } \\
\text { en el curso, ya, puedo estarme imaginando que estoy } \\
\text { haciendo otra cosa. Y, o sea, eso me ayuda bastante a } \\
\text { lo que es imaginarme cosas, de... }\end{array}$ \\
\hline $\begin{array}{l}\text { Capacidad de personificar } \\
\text { personajes y situaciones } \\
\text { mediante la interpretación del } \\
\text { Rol a través del Cosplay. }\end{array}$ & $\begin{array}{l}\text { Ej. [Entrevista]: ...últimamente en Ecuador también ya } \\
\text { se está queriendo incentivar más este tipo de cosas ya } \\
\text { con, como ejemplo en Guayaquil habían hecho una } \\
\text { Comic-Con o algo parecido y ya habían venido así tipo } \\
\text { anime y esas cosas pero aquí, en realidad, está muy } \\
\text { limitado por lo que la mayoría de cosas, digamos, } \\
\text { quedamos con panas cosas que vemos en un anime o } \\
\text { una serie, también, digamos, Game of Thrones y ese } \\
\text { tipo de cosas no venden aquí. }\end{array}$ \\
\hline $\begin{array}{r}\text { Capacidac } \\
\text { los recu }\end{array}$ & $\begin{array}{l}\text { Ej. [Entrevista]: digamos como ya está la capturadora, } \\
\text { se captura todo lo que yo haga y ya puedo jugar. }\end{array}$ \\
\hline $\begin{array}{c}\text { Capacidad de desarrollar } \\
\text { trabajo colaborativo mediante el } \\
\text { uso de diversas plataformas } \\
\text { digitales/comunicación. } \\
\end{array}$ & $\begin{array}{l}\text { Ej. [Entrevista]: Sí, tenemos un grupo. En Facebook } \\
\text { también tenemos un grupo de nuestro curso, entonces } \\
\text { ahí subimos todo lo que hacemos y lo que debemos } \\
\text { hacer o lo que tenemos para esta semana. }\end{array}$ \\
\hline $\begin{array}{l}\text { Capacidad de comunicarse } \\
\text { mediante recursos transmedia. }\end{array}$ & $\begin{array}{l}\text { Ej. [Entrevista]: Ella me dio que vea esa serie, entonces } \\
\text { veo que salen cosas de esa serie, y les comento para } \\
\text { que me manden el enlace de donde ven esa serie. }\end{array}$ \\
\hline $\begin{array}{l}\text { Capacidad para analizar } \\
\text { contenido multimedia. }\end{array}$ & $\begin{array}{l}\text { Ej. [Entrevista]: Por ejemplo, (sustos que dan gusto) veo } \\
\text { esos y luego me imagino como seria lo que sienten ellos } \\
\text { cuando están haciendo eso. }\end{array}$ \\
\hline $\begin{array}{l}\text { Capacidad para identificar y } \\
\text { clasificar contenidos para su } \\
\text { reutilización offline. }\end{array}$ & $\begin{array}{l}\text { Ej. [Entrevista]: era, ver cómo se coge. Hay un, hay una } \\
\text { cosa que se llama Capturadora, se conecta como si } \\
\text { fuera un USB y se le captura la pantalla. Se le prende y } \\
\text { se le captura la pantalla y eso como que tiene una } \\
\text { cámara, le descarga el video y se lo ve. }\end{array}$ \\
\hline $\begin{array}{l}\text { Capacidad de crear recursos } \\
\text { transmedia. }\end{array}$ & $\begin{array}{l}\text { Ej. [Entrevista]: ... tengo unos legos y digamos yo voy } \\
\text { tomando, yo le muevo una piecita y voy tomando cada } \\
\text { parte y luego las uno a todas las partes y sale el stop } \\
\text { Motion. Es como esto [realiza una búsqueda en } \\
\text { YouTube y reproduce un video]. }\end{array}$ \\
\hline $\begin{array}{l}\text { Capacidad de utilizar la } \\
\text { multitarea (eficientemente). }\end{array}$ & $\begin{array}{l}\text { Ej. [Entrevista]: En el buscador de YouTube pone } \\
\text { "música electrónica" y se reproduce y abre otra pestaña } \\
\text { de Google y busca "convertidor mp3" escoge la opción } \\
\text { convertido YouTube a mp3, copia el link del video y lo } \\
\text { pega para descargar y lo comparte en Google }+ \text {. }\end{array}$ \\
\hline
\end{tabular}

Fuente: Los Autores (2019). 


\section{Conclusiones}

En esta investigación se han representado los comportamientos que tienen algunos adolescentes ecuatorianos en su vida cotidiana, manifestados en las prácticas en entornos convergentes y mediáticos. En base a Barreneche, Polo, Menéndez-Echavarría (2018): Ios resultados obtenidos dan cuenta de un comportamiento de los adolescentes en cuanto a las competencias transmedia identificadas y que a su vez han sido evidenciadas en otros estudios (pág. 179). Estos casos analizados describen los perfiles de jóvenes en contextos cambiantes y evidencian las competencias identificadas previamente. Sin embargo, no son evidencias contundentes de una competencia plenamente desarrollada, sino un potencial desarrollo que se puede seguir formando en contextos formales.

Es importante resaltar la naturaleza de los comportamientos de los jóvenes y su inmersión en una cultura digital, a su vez participativa y mediática. Se percibe una tendencia hacia las habilidades transmedia, de remezcla, interpretación de mensajes desde diversas plataformas, que configuran usos, producciones, hábitos y rutinas que han ido desarrollando influenciados en su mayoría por una cultura participativa, de consumo de medios, y a su vez de producción, aunque en menor medida esta última.

Así como, el estudio de Scolari (2017b): sobre el mapeo estrategias informales de uso transmedia en jóvenes de otros contextos (págs. 61-72). El aporte del caso ecuatoriano analizado, evidencia un auge por el consumo masivo de plataformas como YouTube, y el uso de redes sociales como Facebook e Instagram, que ponen de manifiesto un estado de desarrollo de habilidades transmedia para gestionar, buscar información, cambiar de plataformas rápidamente y consumir un mismo mensaje desde diversos medios. Se puede comprobar que existe un nivel intermedio de autorregulación del uso de estos medios, sin embargo, es necesario valorar la influencia que esto está teniendo en los aprendizajes concretos de los jóvenes 
ya que el acceso a la información digital y las redes sociales no se transforma necesariamente en aprendizaje.

Las pedagogías que hacen uso de las estrategias informales, operan a través de líneas participativas de una cultura en línea, de forma que pueden capacitar a los jóvenes, para una amplia gama de recursos educativos digitales para apoyar la colaboración y la creación de conocimientos mediante una pedagogía activa en las aulas escolares de Ecuador.

Este estudio demuestra lo que otros autores han planteado, este es el caso de Fernández y Gutiérrez (2017): sobre el reto de obtener evidencias de aprendizaje informal real y las competencias, y demostrar de qué modo están aprendiendo (pág. 186).

Para futuros estudios, se deja abierto el planteamiento sobre investigar en la aplicación de estas estrategias y sus efectos en los aprendizajes formales, cuestiones que pueden ser indagadas por medio de investigaciones mixtas, que relacionen aspectos cognitivos, psicopedagógicos y mediáticos.

\section{Referencias}

Aparici, R., \& Osuna, S. (2013). La Cultura de la participación. Revista Mediterránea de Comunicación, 4(2), 137-148, e-ISSN: 1989-872X. Recuperado de: http://dx.doi.org/10.14198/MEDCOM2013.4.2.07

Alper, M., \& Herr-Stephenson, R. (2013). Transmedia Play: Literacy Across

Media. JMLE. Journal of Media Literacy Education, 5(2), 366-369. Recovered from: https://digitalcommons.uri.edu/imle/vol5/iss2/2/ Barreneche, C., Polo, N., \& Menéndez-Echavarría, A. (2018). Alfabetismos Transmedia en Colombia: estrategias de aprendizaje informal en jóvenes gamers en contextos de precariedad. Chasqui. Revista Latinoamericana de Comunicación, (137), 169-187, ISSN: 1390-1079; e-ISSN: 1390-924X. Recuperado de:

https://revistachasqui.org/index.php/chasqui/article/view/3510 
Camacho, C. (2018). Visión Teórica Humanística Educativa de la Generación Z 3.0 en Tiempos Complejos. Revista Scientific, 3(9), 2038, e-ISSN: 2542-2987. Recuperado de:

https://doi.org/10.29394/Scientific.issn.2542-2987.2018.3.9.1.20-38

Castells, M. (2001). La galaxia Internet. Reflexiones sobre Internet, empresa y sociedad. Madrid, España: Plaza y Janés Editores, S.A.

Contreras, C., \& León, G. (2019). Análisis factorial de un modelo de socialización y confianza en la dependencia de Internet en estudiantes de secundaria. redie. Revista Electrónica de Investigación Educativa, 21(e25), 1-13, e-ISSN: 1607-4041. Recuperado de:

https://doi.org/10.24320/redie.2019.21.e25.2112

Fernández, N., Blasco-Duatis, M., \& Caldeiro-Pedreira, M. (2016a,b,c). Comunicación y educación en transmedia: Informe sobre competencias en TIC en cuatro centros de educación secundaria de Europa. Cuadernos Artesanos de Comunicación - 108, ISBN 13: 978-84-16458-52-3. Tenerife, España: F. Drago. Andocopias, S.L.

Fernández, E., \& Gutiérrez, J. (2017). La Socialización de los Jóvenes Interconectados: Experimentando la Identidad en la Sociedad Aumentada. Profesorado. Revista de Currículum y Formación del Profesorado, 21(2), 171-190, ISSN: 1138-414X; e-ISSN: 1989-6395. Recuperado de:

https://recyt.fecyt.es/index.php/profesorado/article/view/59455

García-Galera, M., \& Valdivia, A. (2014). Prosumidores mediáticos. Cultura participativa de las audiencias y responsabilidad de los medios. Comunicar, XXII(43), 10-13, ISSN: 1134-3478; e-ISSN: 1988-3293. Recuperado de: https://doi.org/10.3916/C43-2014-a2

Jenkins, H. (2011). Transmedia 202: Further Reflections. Los Ángeles, California, Estados Unidos: Blog de Henry Jenkins. Recovered from: http://henryjenkins.org/blog/2011/08/defining transmedia further re.ht 
$\underline{\mathrm{ml}}$

Jenkins, H., Ford, S., \& Green, J. (2015a,b). Cultura transmedia: La creación de contenido y valor en una cultura en red. ISBN: 9788497848442. Barcelona, España: Editorial Gedisa, S.A.

Jordan, B. (2016). Advancing ethnography in corporate environments: Challenges and emerging opportunities. London, England: Routledge Publisher.

Lerma, L., Cortés, J., \& Barrera, P. (2016). Diagnóstico de la competencia mediática en estudiantes universitarios. RECIE. Revista Electrónica Científica de Investigación Educativa, 3(1), 379-750, ISSN: 1134-3478; e-ISSN: 1988-3293. Recuperado de:

https://rediech.org/ojs/2017/index.php/recie/article/view/278

Livingstone, S., \& Sefton-Green, J. (2016). The Class: Living and Learning in the Digital Age. New York, EE.UU.: University Press.

Masterman, L. (2010). La enseñanza de los medios de comunicación. Volumen 1. Madrid, España: Ediciones de la Torre.

Pereira, Â., Vaz, S., \& Tognetti, S. (2017a,b). Interfaces between science and society. London, England: Routledge Publisher.

Scolari, C. (2013a). Narrativas transmedia: cuando todos los medios cuentan. Barcelona, España: Editorial Planeta.

Scolari, C. (2013b). Media Evolution: Emergence, Dominance, Survival, and Extinction in the Media Ecology. International Journal of Communication, 7, 1418-1441, e-ISSN: 1932-8036. Recovered from: https://ijoc.org/index.php/ijoc/article/view/1919/936

Scolari, C. (2017a,b). Transmedia Literacy: Exploiting transmedia skills and informal learning strategies to improve formal education. Barcelona, Spain: Universitat Pompeu Fabra - UPF. Recovered from: https://repositori.upf.edu/bitstream/handle/10230/33907/Scolari TL fin a.pdf? sequence=1\&isAllowed $=y$ 


\section{Andrea Ximena Castaño Sánchez \\ e-mail: andrea.castano@unae.edu.ec}

Nacida en Pereira, Colombia, el 13 de junio del año 1979.

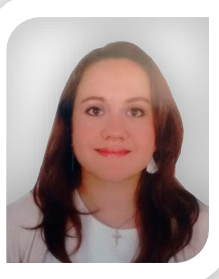

Docente-Investigadora de la Universidad Nacional de Educación (UNAE), en Ecuador; Ingeniera de Sistemas y Computación, Magister en Formación de Formadores; y Doctora en Tecnología Educativa, e-learning y gestión del conocimiento por la Universidad Rovira i Virgili (URV); Con 10 años de experiencia como investigadora y docente en temas relacionados con tecnología y educación, y en proyectos de investigación internacionales; Además, he sido docente en Colombia en maestrías sobre informática y proyectos educativos; y participado como ponente en congresos internacionales en temas sobre portafolios digitales en educación superior. 


\section{Manuel Torres Mendoza \\ e-mail: mtorres@upse.edu.ec}

Nacido en Guayaquil, Ecuador, el 7 de julio del año

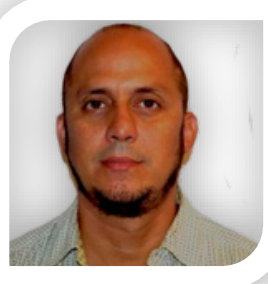

1972. Docente-Investigador; Coordinador de la Maestría de Comunicación; y Director del Observatorio Turístico Sostenible en la Universidad Estatal Península de Santa Elena (UPSE); Docente y tutor de proyectos de la Maestría en Social Media de la Universitat Oberta de Catalunya (UOC); Docente invitado en las Maestrías de Comunicación y la de Periodismo Digital de la Universidad Casa Grande (UCG), de Guayaquil; He impartido desde el año 2005 al 2010 en toda Catalunya, centenares de talleres a jóvenes sobre el uso responsable de las TIC, la estructura de los medios y el consumo responsable; También investigo sobre los factores que intervienen en la construcción social de la realidad y la configuración del imaginario colectivo a través de distintos soportes y redes de influencia. 


\section{Marcos Manuel Ibarra Núñez}

e-mail: marcos.ibarra@unae.edu.ec

Nacido en Zacatecas, México, el 9 de mayo del año 1987.

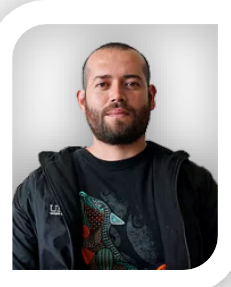

Experto en el área de tecnología y educación; Experiencia en el campo de la Robótica pedagógica; Dominio de estrategias y metodologías didácticas para la enseñanza con apoyo de las TIC; Docente del área de mecatrónica en nivel superior (5to año), en diversas asignaturas como cálculo, control automático, diseño asistido por computadora, electrónica analógica, instrumentación virtual, robótica industrial, entre otras. Trabajo con entornos virtuales de aprendizaje, desarrollo de contenidos y virtualización de los mismos; Desarrollo de recursos didácticos digitales; Dominio en las teorías pedagógicas actuales, vinculadas a las TIC; Docente de didáctica de las matemáticas; Especialista en el tema de discapacidad e inclusión; Actualmente docente investigador de la Universidad Nacional de Educación (UNAE).

El contenido de este manuscrito se difunde bajo una Licencia de Creative Commons ReconocimientoNoComercial-Compartirlgual 4.0 Internacional 